\title{
GPS Landing System Reference Antenna
}

\author{
Alfred R. Lopez \\ BAE Systems - Electronics, Intelligence and Support \\ 450 Pulaski Road, Greenlawn, NY 11740 USA \\ Tel: +1 (631) 262-8021; Fax: +1 (631) 262-8053; E-mail: al..lopez@baesystems.com
}

\begin{abstract}
The GPS landing system (GLS) is a reality today, and will undoubtedly become the workhorse system in the future. GPS aircraft navigation is currently utilized for aircraft en-route, terminal, and initial-approach navigation. It is expected that in 2009, a Category I GPS landing system will start its initial phase of a worldwide deployment. The ARL-1900 antenna was designed specifically to satisfy the stringent requirements for the Category I, II, and III GPS landing system reference-receiver stations.

A difficult problem for a Category I, II, and III GPS landing systems is the mitigation of ground-reflected multipath effects. The antenna must provide coverage of the upper hemisphere while suppressing ground-reflected multipath. In addition, the antenna must operate at the L1, L2, and L5 GPS frequencies, have right-hand circular polarization, and ideally have constant carrier and code (group) delay throughout the coverage region. Over a period of 15 years, BAE Systems has developed the ARL-1900 antenna, a unique antenna with near-ideal performance that satisfies the stringent requirements for a Category I, II, and III GPS landing systems. This paper reviews the requirements for a GPS landing system reference antenna, presents the design principles for the ARL-1900 antenna, describes its implementation, and presents performance data.
\end{abstract}

Keywords: Antenna arrays; Global Positioning System; GPS reference antenna; delay effects; GPS antenna group delay; GPS antenna carrier delay; wideband GPS antenna

\section{Introduction}

$\mathbf{T}$ he instrument landing system (ILS) was introduced in 1941. It was selected by the ICAO (International Civil Aviation Organization) in 1946 as the international all-weather landing aid [1]. It is currently the primary worldwide aircraft landing system. It uses a localizer antenna to provide horizontal guidance with respect to the runway's centerline, and a glide-slope antenna to provide vertical guidance with respect to the runway's glide path. Distance-to-runway-threshold guidance is provided by marker beacon antennas and/or a DME (distance measuring equipment) antenna.

The microwave landing system (MLS) was originally intended to replace the instrument landing system. The widespread deployment initially envisioned by its designers in the 1970s and 1980s never became a reality. GPS-based landing systems (GLS), notably WAAS (wide-area augmentation system), provide the same level of positional accuracy and substantially larger coverage, with no equipment needed at the airport. The dramatically lower cost and performance advantages of a wide-area-augmentation-system GPS landing system have led to the turning off of most existing microwave landing systems in North America. The microwavelanding-system mode of operation is basically the same as that of the instrument landing system. A microwave-landing-system azimuth antenna provides horizontal guidance with respect to the azimuth approach path, an elevation antenna provides vertical guidance with respect to the elevation approach path, and a distance-measuring-equipment antenna provides guidance with respect to the distance from the runway's threshold. However, the antenna technology is much different. A microwave landing system incorporates electronically scanned azimuth and elevation antennas operating at a much higher frequency, which provides significant performance advantages over an instrument landing system.

The operation of the GPS aircraft navigation system is similar to that of the ubiquitous car GPS navigator. This has two key elements: a GPS receiver, including an antenna; and a geographical information system (GIS). The geographical information system integrates hardware, software, and data, and provides output data to the driver. In concept, the geographical information system's memory stores the address (name), latitude, and longitude for each point within the GPS navigator's coverage region. The GPS receiver provides the geographical position (latitude and longitude) of the car as input to the geographical information system. The driver's input is the address of the desired destination. The geographical information system analyzes the GPS receiver and driver inputs, determines a route for the car to follow, and provides visual and audio outputs to aid the driver in reaching the desired destination. GPS aircraft navigation uses the three coordinates - latitude, longitude, and altitude - for establishing the aircraft's position. 
The US FAA (Federal Aviation Agency) expects to utilize GPS aircraft navigation for all phases of flight: en-route, terminal, approach, and surface navigation. An unaided GPS navigation system can satisfy the requirements for en-route operations. The wide-area-augmentation-system GPS landing system satisfies the requirements for terminal- and initial-approach operations. The LAAS/GBAS (local-area augmentation system/ground-based augmentation system) GPS landing system will satisfy the requirements for final approach. Initially, a local-area augmentation system will augment GPS to improve aircraft safety during Category I airport approaches and landings. Ultimately, a local-area augmentation system will provide the high accuracy, availability, and integrity necessary for Category II and III precision approaches.

As shown in Figure 1, the local-area augmentation system incorporates four reference receiver (RR) stations to determine the broadcast corrections for the satellite-to-aircraft distances [2]. Each reference-receiver station has a reference antenna. Signals from GPS satellites are received by the local-area augmentation system reference receivers. The reference receivers calculate their position using GPS. The reference receivers and local-area augmentation system ground facility (LGF) work together to measure errors in

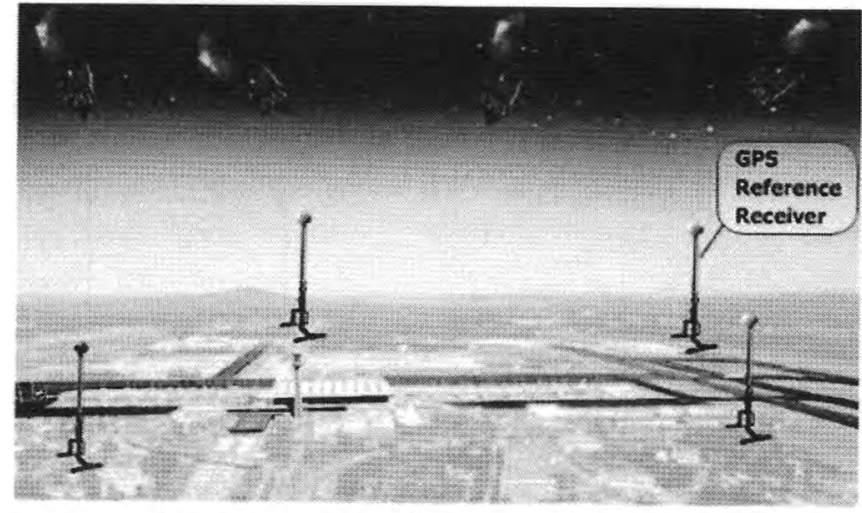

GPS Reference Receivers Calculate Position

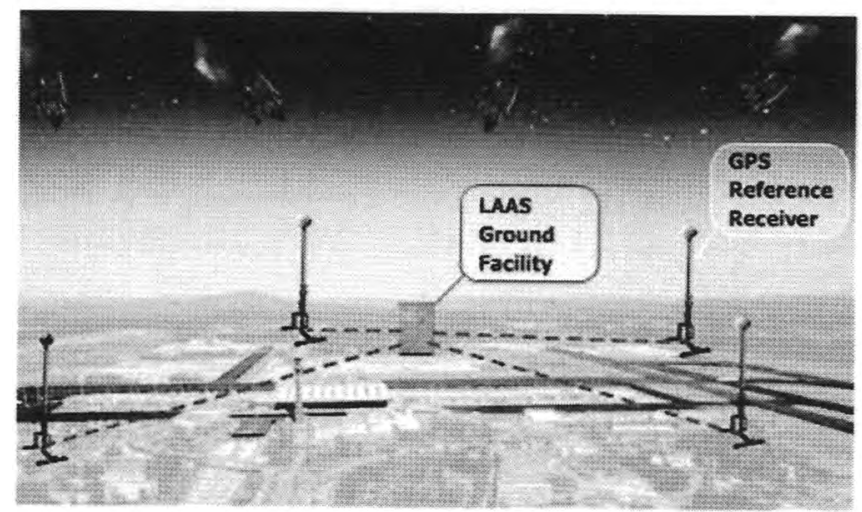

LAAS Ground Facility (LGF) Calculates Errors in GPS Position and Formulates the LAAS Correction Message the GPS-provided position. The local-area augmentation system ground facility produces a local-area augmentation system correction message based on the difference between the actual and GPScalculated reference-receiver position. Included in this message are suitable integrity parameters and approach-path information. The local-area augmentation system correction message is then sent to a VHF data broadcast (VDB) transmitter. The VHF data broadcast transmitter broadcasts the correction message to aircraft equipped with the local area augmentation system in the service area. The aircraft uses the broadcast corrections to achieve the required positioning accuracy for precision approach and landing.

The reference-receiver subsystem is not necessarily restricted to four reference receivers. The system-error component attributed to the reference-receiver subsystem is inversely proportional to the square root of the number of reference receivers. The number four was selected based on redundancy, accuracy, and cost considerations. The two main error components for a reference receiver are receiver thermal noise and multipath. Receiver post filtering is used to reduce the reference-receiver thermal-noise rms error component to $0.05 \mathrm{~m}$. The dominant multipath component is ground multipath. The four reference receivers are spaced so that their

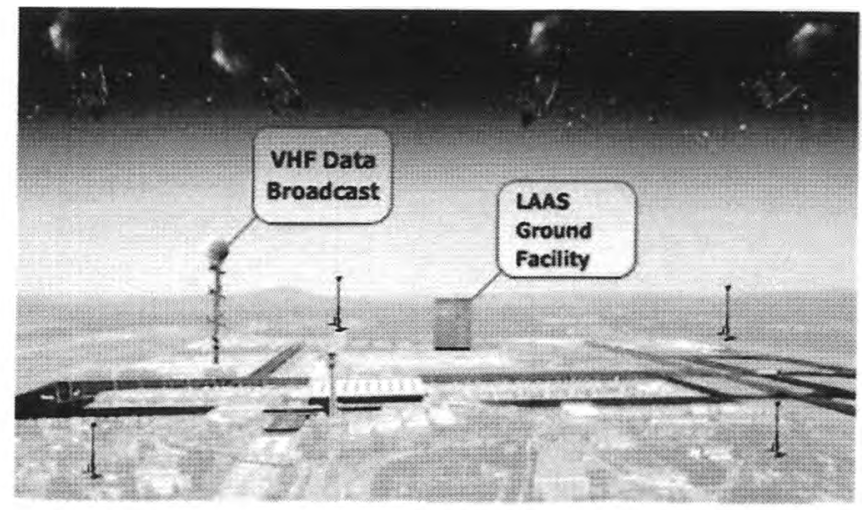

The LAAS Ground Facility Sends the Correction Message to the VHF Data Broadast (VDB) Transmitter

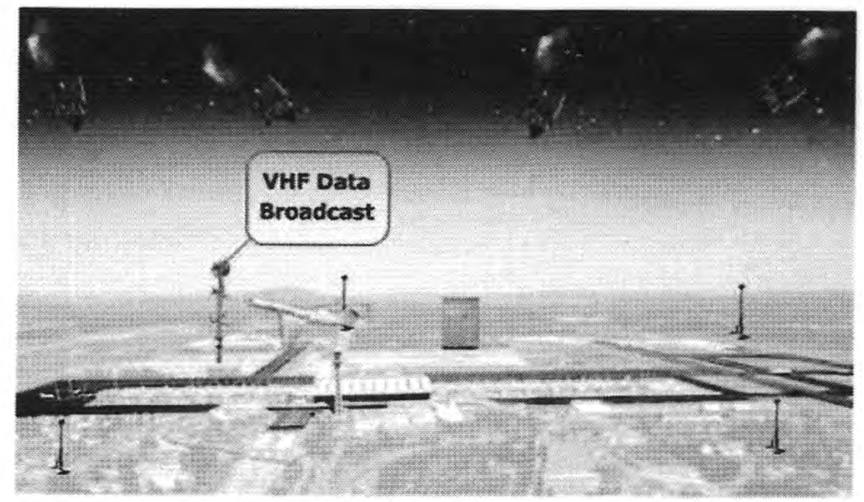

The VDB Transmitter Broadcasts the LAAS Signal to Equipped Aircraft in the Service Area

Figure 1. The operation of the local-area augmentation system (top to bottom): The GPS reference receivers calculate position; the local-area augmentation system ground facility calculates errors in GPS position and formulates the local-area augmentation system correction message; the local-area augmentation system ground facility sends the correction message to the VHF data broadcast transmitter; the VHF data broadcast transmitter broadcasts the local-area augmentation system signal to equipped aircraft in the service area. 
ground-multipath errors are decorrelated. Multipath error can be mitigated with a well-designed reference-receiver antenna. The design objective for the ground-reference antenna is to limit the rms multipath error to $0.05 \mathrm{~m}$. The ARL-1900 antenna (Figures 2 and 3 [3]) was developed specifically to satisfy the stringent antenna requirements for the local-area augmentation system GPS landing system. This paper discusses the antenna requirements, presents the basic antenna-design principles, describes the implementation of the ARL-1900 antenna, and presents antenna performance data.

\section{Antenna Requirements}

\subsection{Angular Coverage}

The antenna has to receive signals from satellites in the upper hemisphere for all azimuth angles and elevation angles ranging from $5^{\circ}$ to $90^{\circ}$.

\subsection{Frequency}

The current local-area augmentation system operates at the $\mathrm{L} 1$ frequency $(1575.42 \mathrm{MHz})$. In the future, it is expected that the local-area augmentation system will also operate at the L2 frequency $(1227.6 \mathrm{MHz})$ and the L5 frequency $(1176.45 \mathrm{MHz})$. Ideally, the ground reference antenna should operate over all the frequencies corresponding to the satellite constellations that will be part of the Global Navigation Satellite System (GNSS).

\subsection{Polarization}

The GPS satellites transmit right-hand circular polarization (RHCP). The ideal polarization for the ground reference antenna is therefore RHCP in every direction within the coverage region. (RHCP down to $0^{\circ}$ elevation angle also provides suppression of errors caused by lateral (non-ground) multipath [4].)

\subsection{Gain}

Ideally, the gain should be uniform within the coverage region. The gain requirements are presented in Table 1.

\subsection{Radiation Pattern Shape for Ground Multipath Suppression} are:

The requirements for the elevation-angle radiation pattern

- 6-dB beamwidth: $174^{\circ}$

- Lower-hemisphere sidelobe level $\left(<-5^{\circ} \mathrm{EL}\right)$ :

$\geq 30 \mathrm{~dB}$ below peak
- Gain slope at the horizon: $\geq 2.5 \mathrm{~dB} /{ }^{\circ}$

Figure 2 shows the computed and measured radiation patterns. These requirements insure that the ground-multipath rms error will be less than $0.090 \mathrm{~m}$ when the antenna is operating over a ground with the worst-case reflectivity (smooth sea water) [3].

\subsection{Carrier Delay and Code (Group) Delay Variations}

The GPS signal's carrier delay and code delay are related to each other, but they are not necessarily equal to each other [5]. The GPS signal's carrier-delay and code-delay variations within the coverage region must not exceed specified limits for establishing the antenna's spatial-physical reference point (the antenna's phase center: see the Appendix for the definition of the antenna's phase center), used in defining the reference-receiver position. If the limits are exceeded, then an antenna calibration procedure [5] must be used to determine the reference receiver's position within the required error tolerance. (The ideal antenna has zero carrier-delay and zero code-delay variations within the coverage region.) The requirements for carrier delay and code delay with respect to the spatial reference point (typically, near the center of the antenna), and within the coverage region, are as follows:

$\begin{array}{ll}\text { - rms carrier-delay variation: } & \leq 0.007 \mathrm{~m} \\ \text { - rms code-delay variation: } & \leq 0.025 \mathrm{~m}\end{array}$

\subsection{Lightning Protection}

FAA-STD-019d [6] requires that air terminals be placed so as to protect antennas mounted on towers. The air terminal should have a minimal effect on antenna performance.

\section{ARL-1900 Design Principles}

The sharp cutoff $[7,8]$ requirement for the elevation-angle radiation pattern, and the omnidirectional azimuth-angle radiation pattern required for the coverage of the upper hemisphere, led to a vertical collinear array antenna as the sole viable solution for this set of antenna requirements. The radiation pattern of an array antenna can be expressed as the product of two factors: the array factor and the element factor. The product is strictly correct for an infinite array, where all elements have the same environment. The ARL-1900 antenna has 19 elements. The bottom two elements

Table 1. The gain requirements for the local-area augmentation system GPS landing system reference antenna.

\begin{tabular}{|c|c|c|}
\hline $\begin{array}{c}\text { Frequency } \\
\text { (MHz) }\end{array}$ & $\begin{array}{c}\text { Minimum Gain at } \\
\mathbf{5}^{\circ} \text { Elevation Angle } \\
\text { (dBiRHCP) }\end{array}$ & $\begin{array}{c}\text { Typical Gain } \\
\text { Within Coverage } \\
\text { (dBiRHCP) }\end{array}$ \\
\hline L1 (1575.42) & -6.0 & -3.0 \\
\hline L2 (1227.60) & -8.0 & -4.0 \\
\hline L5 (1176.45) & -9.0 & -4.5 \\
\hline
\end{tabular}



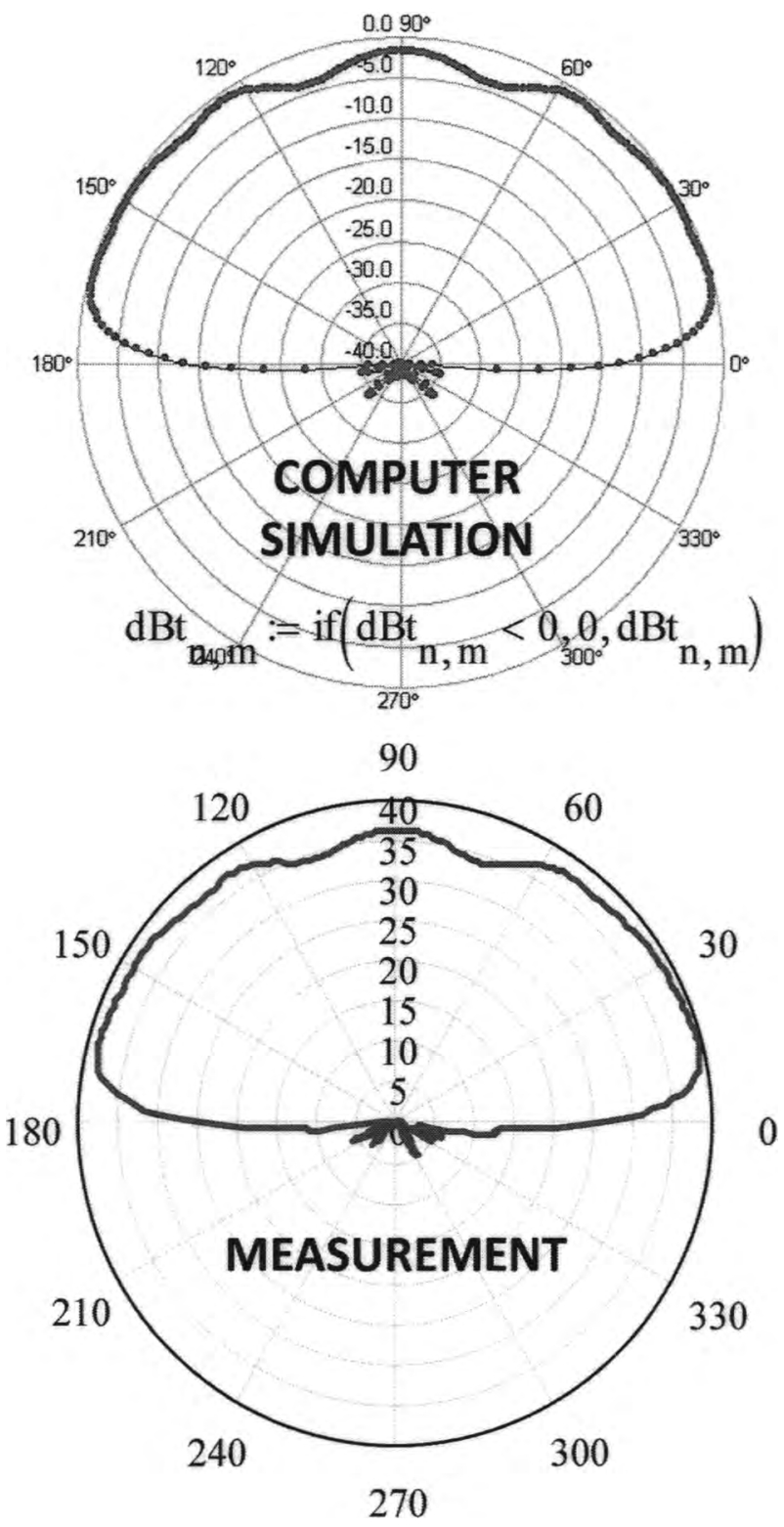

Figure 2a. The computed (top) and measured (bottom) antenna patterns for the ARL-1900 antenna.

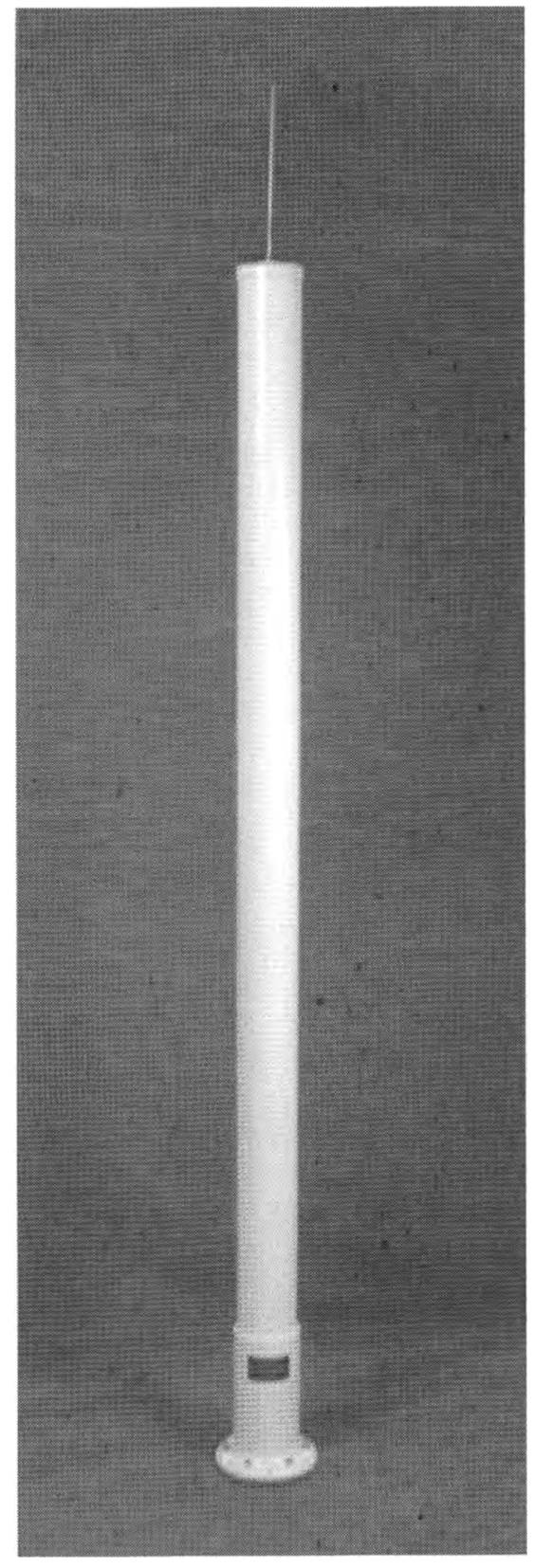

Figure 2b. The BAE Systems ARL-1900 GPS landing-system reference antenna

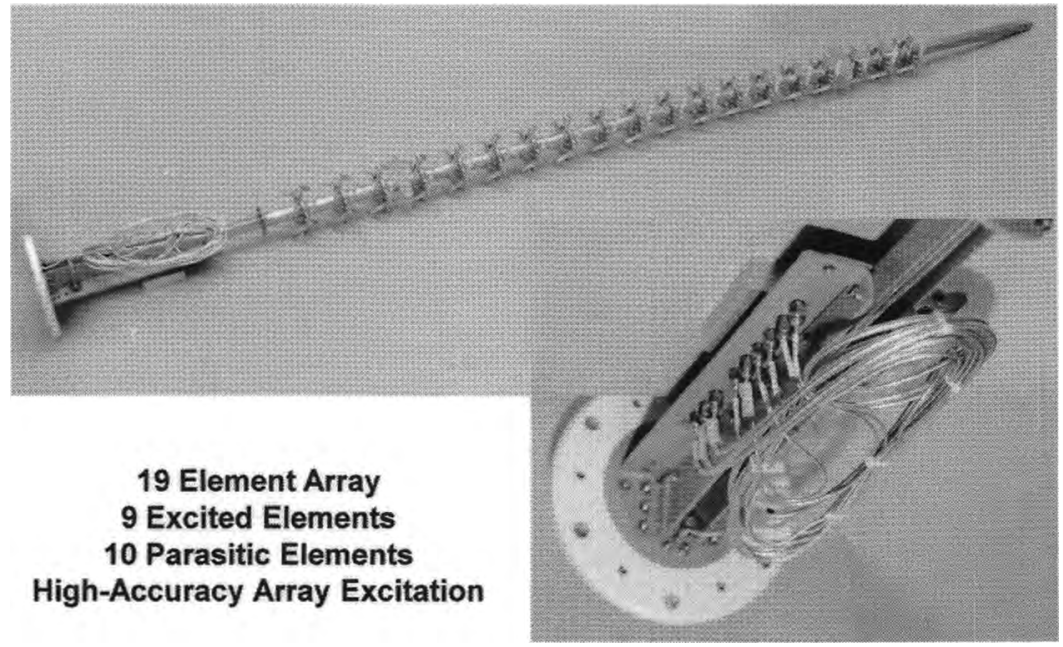

Figure 3. The ARL-1900 antenna with the radome removed. 
(first and second) and the top two elements (18th and 19th) are called dummy (parasitic) elements because they are not directly excited. Their function is to provide a near-infinite-array environment for the third and 17th elements, which are the array's edge elements, and are excited. The vertical extent of the array in wavelengths must be sufficiently large enough to provide the required sharp cutoff characteristics near the horizon.

\subsection{Array Element}

The array-element design is crucial to the overall performance of the array. It controls the polarization and impedance matching. It can also affect the quality of the antenna's phase center, and the coverage of the array antenna. The ARL-1900 array element has four slanted dipoles that are fed by a four-way power divider, which provides equal power and $0^{\circ}, 90^{\circ}, 180^{\circ}$, and $270^{\circ}$ phase to the dipoles. The element in the array environment has right-hand circular polarization in the upper hemisphere, horizontal linear polarization near $-30^{\circ}$ elevation angle, and left-hand circular polarization in the nadir direction. The element gain pattern is close to being isotropic.

The impedance-matching objective was to minimize internal reflections between the element connectors and the power-divider connectors. This was achieved by impedance matching the element in the array environment. The VSWR at the dipole's feed point was less than 2:1 for the L1, L2, and L5 frequencies.

\subsection{Array Factor}

A basic equation for the radiation pattern of a vertical collinear array antenna is

$$
E(\theta)=e(\theta) A F(\theta)
$$

where $E(\theta)$ is the array element's pattern, $e(\theta)$ is the element pattern, and $\theta$ is the pattern angle. The array factor pattern, $A F(\theta)$, is given by

$$
A F(\theta)=\sum_{n=-\frac{N-1}{2}}^{n=\frac{N-1}{2}} a_{n} e^{j 2 \pi n \frac{d}{\lambda} \sin (\theta)}
$$

where $n$ is the element number, $a_{n}$ is the element amplitude and phase excitation, $d$ is the element spacing, $\lambda$ is the free-space wavelength, and $N$ is the total number of array elements ( $N$ is odd). It should be noted that $E(\theta)$ is valid if all elements are identical and there is a sufficient number of dummy elements such that pattern-factor multiplication is valid.

The ARL-1900 is inherently a low-gain antenna. An ideal antenna that covers a hemisphere has a peak gain of $3 \mathrm{dBi}$. It is very difficult to achieve low sidelobes with a low-gain antenna. The array-excitation amplitude and phase errors create a noise-like radiation component that is spread uniformly in angle. The rms excitation-error sidelobe level relative to the beam peak is given by

$$
S L_{E E}=\sqrt{\frac{\frac{\delta A^{2}+\delta P^{2}}{3}}{G\left(1-\frac{\delta A^{2}+\delta P^{2}}{3}\right)}},
$$

where $S L_{E E}$ is the rms excitation-error sidelobe level (voltage ratio), $\delta A$ is the peak amplitude error (voltage ratio), $\delta P$ is the peak phase error (radians), and $G$ is the antenna's directive gain (power ratio, $G=2$ for hemispherical coverage).

It is clear from the above equation that for a given error tolerance, the excitation-error sidelobe power level is inversely proportional to the antenna's power gain. Higher-gain antennas will have a lower excitation-error sidelobe level. Figure 4 presents the twosigma excitation-error sidelobe level as a function of the amplitude error and phase error for a $3 \mathrm{dBi}$-gain antenna.

The array factor near-peak sidelobe level is given by

$$
S L_{A F}=\sqrt{\left(2 S L_{E E}\right)^{2}+S L_{Z E}^{2}}
$$

where $S L_{A F}$ is the array-factor near-peak sidelobe level (voltage ratio), $S L_{E E}$ is the rms excitation-error sidelobe level (voltage ratio), and $S L_{Z E}$ is the peak zero-error sidelobe level (voltage ratio).

A strategy for achieving near $30 \mathrm{~dB}$ sidelobes is to design for $36 \mathrm{~dB}$ peak zero-error sidelobes and $30 \mathrm{~dB}$ two-sigma excitationerror sidelobes. The resulting array-factor pattern has sidelobes that are dominated by the excitation-error sidelobes, and are predominantly random amongst a group of several antennas. This feature enhances the desired decorrelation of the four local-area-augmentation-system reference receivers.

Figure 5 shows the results of a Monte Carlo simulation of the ARL-1900 antenna array factor. These results were in good agreement with those predicted by Equations (2) and (3). When converted to the two-sigma value (Figure 4), Equation (2), predicted that the two-sigma excitation-error sidelobe level should be

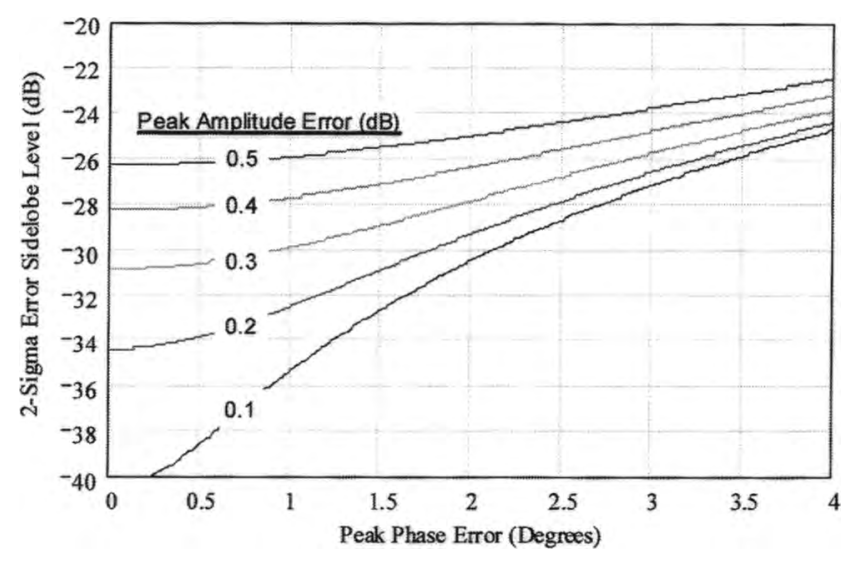

Figure 4. The two-sigma excitation-error sidelobe level of the array antenna as a function of the peak phase error, with the peak amplitude error as a parameter. A $3 \mathrm{dBi}$ gain antenna was assumed. 


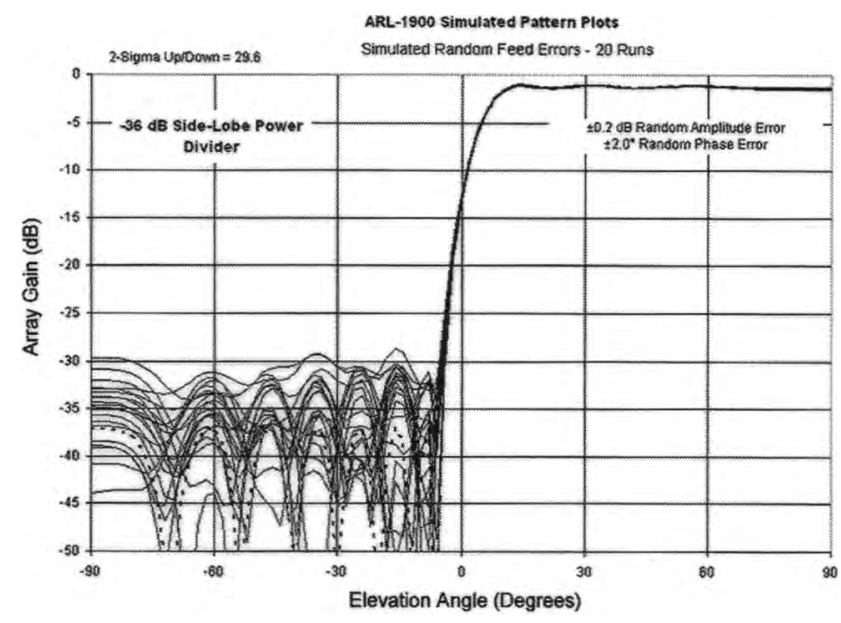

Figure 5. The array-factor pattern for 20 trials with random errors of $\pm 0.20 \mathrm{~dB}$ amplitude and $\pm 2.0^{\circ}$ phase. The dotted curve is the $-36 \mathrm{~dB}$ zero-error peak sidelobe level.

$-29.3 \mathrm{~dB}$ for $\pm 0.20 \mathrm{~dB}$ amplitude and $\pm 2.0^{\circ}$ phase errors. The root sum square of the $-29.3 \mathrm{~dB}$ two-sigma excitation-error level and the $-36 \mathrm{~dB}$ zero-error sidelobe level was $-28.5 \mathrm{~dB}$. The peak sidelobe level shown in Figure 5 was $-28.0 \mathrm{~dB}$.

\subsection{Carrier Delay and Code (Group) Delay}

For the measurement of distance between a satellite and the reference-receiver antenna, the antenna's spatial reference point (phase center) should ideally have a constant-carrier-delay (phase) pattern over the coverage region. For this ideal case, the code-delay pattern over the coverage region is also a constant value when measured with respect to the same point.

The array-factor excitation that is used for the ARL-1900 (see [8], Table 1 , for a representative excitation) produces an arrayfactor pattern that has constant carrier delay and constant code delay in the upper hemisphere. It has the ideal carrier-delay and code-delay patterns. The phase center is at the center of element 10 , the center element. In practice, because of mutual coupling among the array elements, the signal in the zenith direction is delayed about $4.5 \mathrm{~cm}$ (see Figure $8 \mathrm{a}$ ). This lowers the antenna phase center so that it is located $4.5 \mathrm{~cm}$ below the center element. The resulting computed and measured carrier-delay patterns are shown in Figure 8. The carrier delay variation was $\pm 1.0 \mathrm{~cm}$.

\subsection{Lightning Protection}

The lightning-protection system for the ARL-1900 consists of three basic components: (1) an air terminal; (2) a down conductor; and (3) two electrical-bonding devices: an air-terminal-to-downconductor bonding device, and a down-conductor-to-base-plate bonding device. The air terminal is 12 in long, and has a diameter of $3 / 8 \mathrm{in}$. It is a nickel-plated solid-copper conductor. The down conductor is tin plated AWG 2 stranded copper. The bondingdevice material is aluminum alloy, and has two stainless-steel setscrews that connect the bonding device to the down conductor. The down conductor passes through the center of the radiating ele- ments, and connects the air terminal at the top of the antenna to the base plate, which is grounded.

The air terminal is perpendicular to the electromagnetic-field vector, and consequently is not excited by the electromagnetic field. There is no electromagnetic-field scattering caused by the air terminal. Theoretically, it has no effect on the antenna's performance.

The ARL-1900 antenna shown in Figure 2 has the air terminal attached at the top of the radome.

\section{ARL-1900 Physical Description}

As shown in Figure 3, the ARL-1900 has 19 array elements, nine of which are excited by nine coaxial cables that are connected to a nine-way power divider. The power-divider/cabling assembly provides equal electrical path length from the power divider's input connector to the nine output coaxial connectors that attach to element numbers $3,5,7,9,10,11,13,15$, and 17 (element 1 is the bottom element). The other 10 elements operate as parasitic elements, and are essential for the operation of the antenna.

The air terminal is attached at the top of the antenna. The down conductor threads through the elements, and is bonded to the base plate. The power divider is mounted to the central support structure, just above the base plate. The excess cabling is wound in a coil to fit within the radome. The radome material is G-10 fiberglass. The outside diameter of the radome is $102 \mathrm{~mm}$ (4.0 in). The overall height of the antenna, including the air terminal, is $2.3 \mathrm{~m}$ $(7.6 \mathrm{ft})$. The weight of the antenna is $9.5 \mathrm{~kg}(21 \mathrm{lb})$.

\section{ARL-1900 Performance}

\subsection{Gain and Polarization}

The measured gain for the ARL-1900 antenna satisfies the requirements of Table 1 . The measured polarization performance is presented in Table 2 .

\subsection{Radiation Patterns}

WIPL-D antenna CAD software was used in the early development of the ARL-1900 antenna. It provided a high level of confidence in the design approach. (Antenna CAD is an evolving art. For the ARL-1900 antenna, a complete computer simulation was not feasible. Not included in the simulation was the four-way power divider incorporated in each of the array elements. It was

Table 2. The measured polarization axial ratio for the ARL-1900 antenna.

\begin{tabular}{|c|c|c|}
\hline \multirow{2}{*}{$\begin{array}{c}\text { Frequency } \\
\text { (MHz) }\end{array}$} & \multicolumn{2}{|c|}{ Axial Ratio (dB) } \\
\cline { 2 - 3 } & EL Angle, $\mathbf{0}^{\circ}$ to $\mathbf{1 0}^{\circ}$ & EL Angle, $\mathbf{1 0}^{\circ}$ to $\mathbf{9 0}$ \\
\hline \hline L1 $(1575.42)$ & $<3$ & $<6(4$ typical $)$ \\
\hline L2 $(1227.60)$ & $<10$ & $<7(4$ typical $)$ \\
\hline L5 $(1176.45)$ & $<10$ & $<7(4$ typical $)$ \\
\hline
\end{tabular}


later determined that the four-way power divider does have some secondary effects, mainly in the cross-polarized pattern.) Figure 6 presents a comparison of the early WIPL-D predictions and the measured radiation pattern. The patterns were measured at the BAE Systems Greenlawn, NY, indoor antenna test range. The agreement between the early WIPL-D predictions and the later measurements was excellent. Figure 7 shows the measured gain patterns for the L1 and L2 frequencies.

The measurements presented in Figure 6 were made on antenna serial No. 026, which was a quality-assurance test specimen. It was subjected to a stringent set of environmental tests (wind, rain, vibration, salt fog, temperature, humidity, altitude, and hail). The radiation pattern shown in Figure 6 was obtained during the acceptance-test-procedure (ATP) testing, following the qualityassurance testing. In general, there was very little difference in the acceptance-test-procedure test results before and after the qualityassurance testing. These results provided confidence in the soundness of the electrical and mechanical designs.

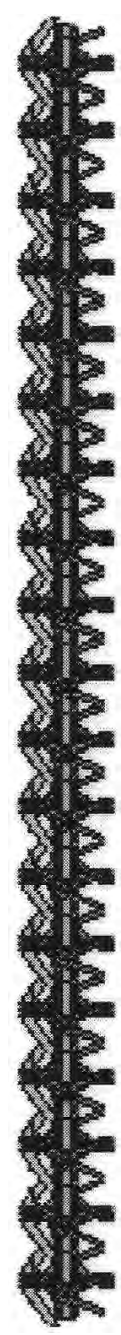

Figure 6a. The WIPL-D model.

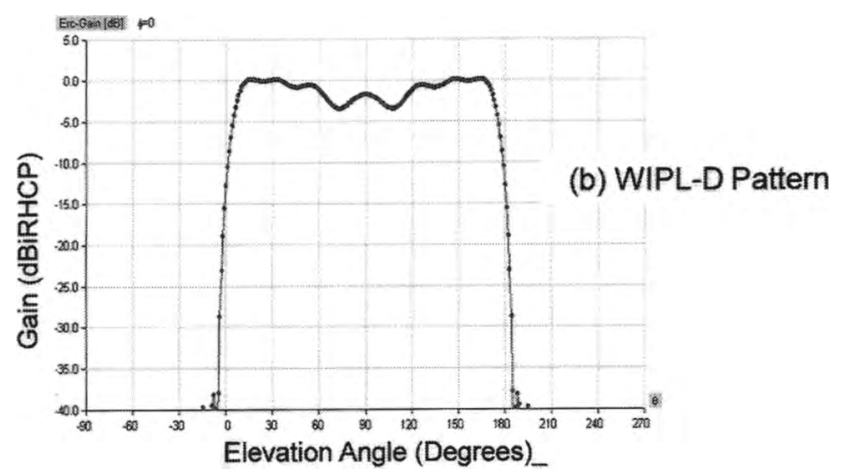

Figure 6b. The computed gain pattern (dBiRHCP).

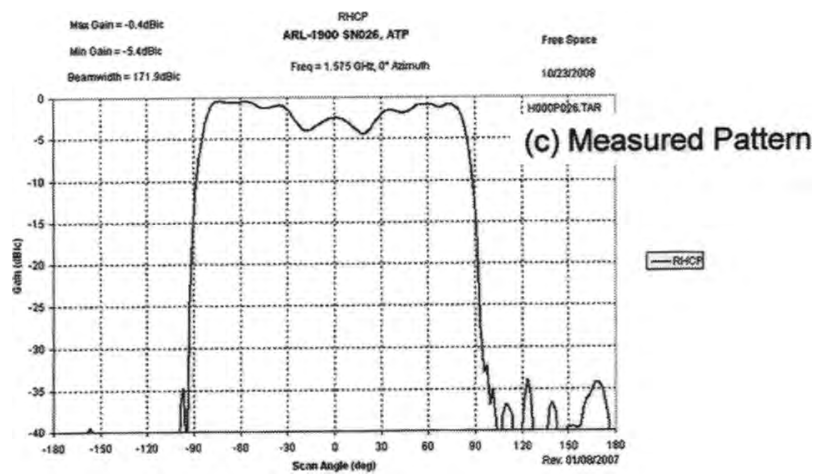

Figure 6c. The measured gain pattern (dBiRHCP).

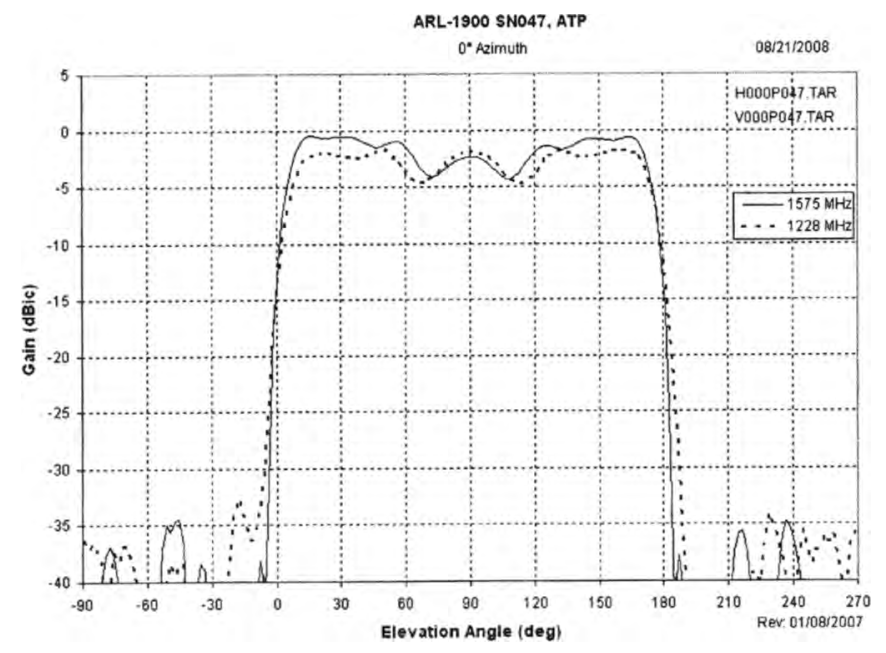

Figure 7. The measured gain patterns (dBiRHCP) for the L1 and $L 2$ frequencies.

\subsection{Carrier Delay and Code (Group) Delay}

Figure $8 \mathrm{~b}$ presents the measured carrier delay as a function of elevation angle for 16 antennas (serial Nos. 003-018). The measurements were made at the BAE Systems indoor antenna range in Greenlawn, NY. The phase center (the phase-reference point that minimizes the carrier-delay variation over the coverage region) was located $4.5 \mathrm{~cm}$ below the center element. The carrier-delay variation with elevation angle was $\pm 0.010 \mathrm{~m}$. The consistency among the 16 antennas at any elevation angle was $\pm 0.002 \mathrm{~m}$. 
It is very difficult to measure code delay in a conventional indoor antenna range [5]. A methodology has been developed for measuring code-minus-carrier (CMC) delay using the GPS satellite constellation $[5,9]$. The code delay is then determined from

$$
\text { Code }=\text { Code-Minus-Carrier }+ \text { Carrier } \text {. }
$$

Figure 9 presents the results of measurements performed on four ARL-1900 antennas at the FAA Tech Center, Atlantic City, NJ. The code-minus-carrier delay bias (mean) and code-minus-carrier delay standard deviation (STD) for the four antennas were evaluated over a period of 24 hours. Each point was the result of an averaging or standard-deviation process of code-minus-carrier delay evaluated for all satellites in a $2^{\circ}$ elevation-angle bin. The bias component was characteristic of the antenna for elevation angles below $60^{\circ}$. Because of the scarcity of data points, at the higher elevation angles multipath can affect the bias value. The standard deviation has receiver noise and multipath components. For the ARL-1900, receiver noise was the dominant standarddeviation component. The thick-line curve in Figure 9 was an estimate of the ARL-1900 antenna's code-minus-carrier delay variation with elevation angle.

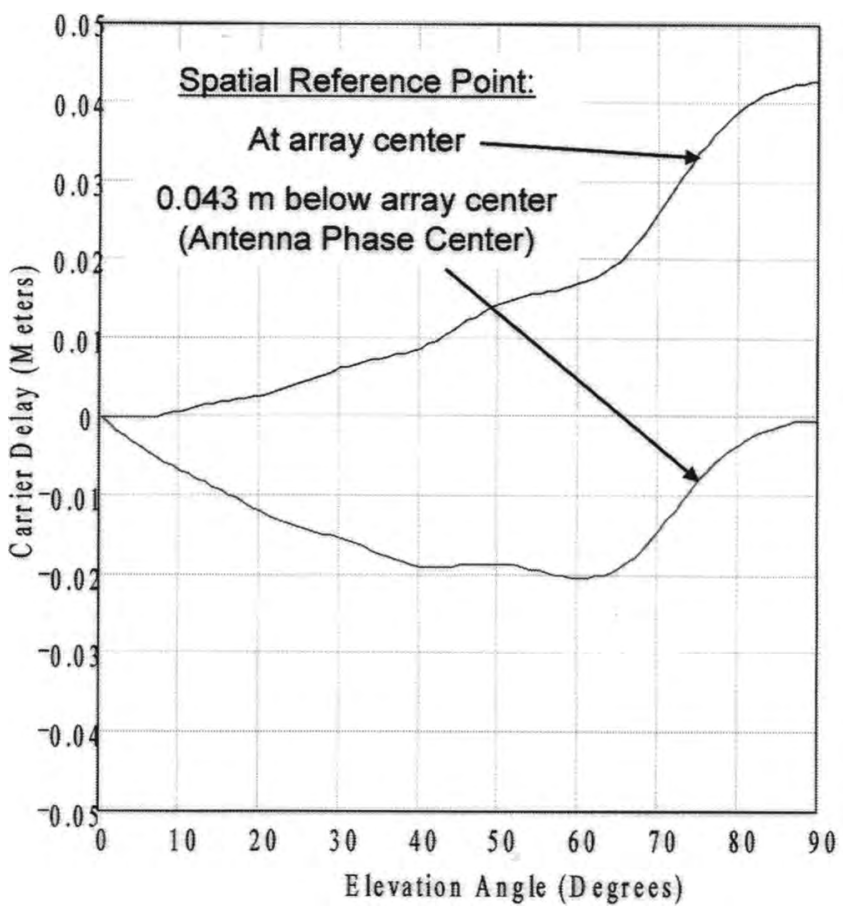

Figure 8a. The WIPL-D computed carrier delay as a function of elevation angle.

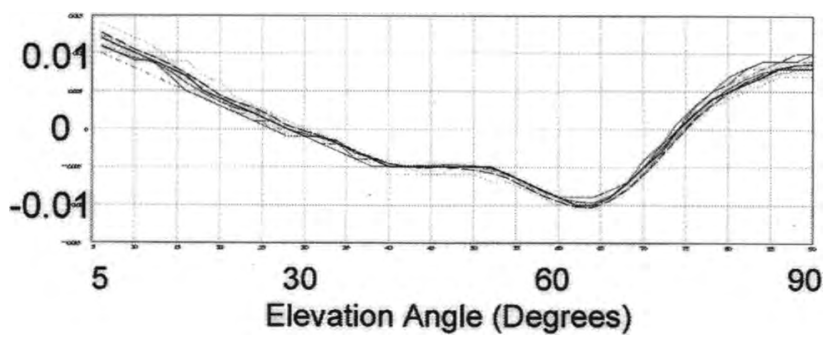

Figure 8b. The measured carrier delay as a function of elevation angle. The data shown were for 16 antennas, with phase center located $0.045 \mathrm{~m}$ below the array's center.

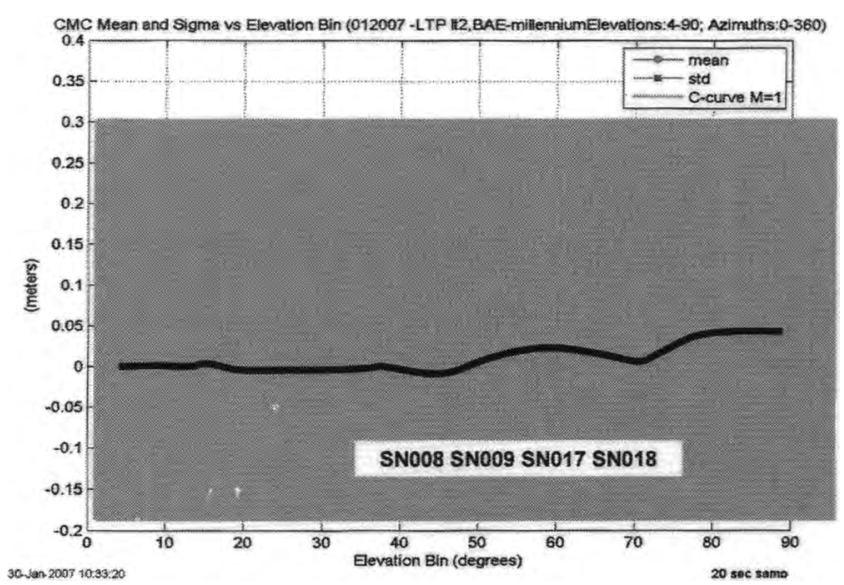

Figure 9. Code-minus-carrier measurements made at the FAA Tech Center, January 2007.

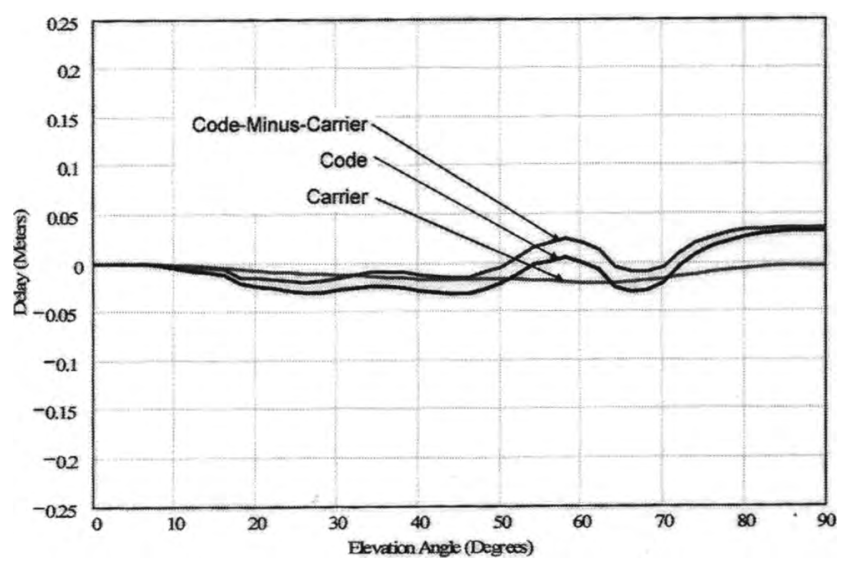

Figure 10. The code-minus-carrier, carrier, and code delay as a function of elevation angle. The code-minus-carrier delay was the average of measurements for nine antennas. The carrier delay was the average of measurements for 16 antennas.

Figure 10 shows code-minus-carrier delay, carrier delay, and code delay as functions of elevation angle for the ARL-1900 antenna. The code-minus-carrier delay was determined from FAA measurements of nine antennas. Carrier delay was determined from BAE Systems' measurement of 16 antennas. The code delay was determined by the use of Equation (4). It was estimated that the rms code-delay variation over the range of elevation angles of $5^{\circ}$ to $90^{\circ}$ was $0.020 \mathrm{~m}$.

\section{Summary}

The GPS landing system will undoubtedly become the workhorse system in the future. GPS aircraft navigation is currently utilized for aircraft en-route, terminal, and initial-approach navigation. It is expected that in 2009, the Category I local-area augmentation system/ground-based augmentation system GPS landing system will start its initial phase of a worldwide deployment. The ARL-1900 antenna was designed specifically to satisfy the stringent requirements for the local-area augmentation system/groundbased augmentation system GPS landing system reference-receiver stations. 
- One port, circular polarization, L1-L2-L5 coverage of the upper hemisphere

- One port, one receiver, and no satellite-handover problem. (The original local-area augmentation system reference-receiver station used two integrated antennas. The integrated antenna was manufactured by dB Systems Inc. It had two ports (one for each antenna), two receivers were required to cover the upper hemisphere, and it operated only at the L1 frequency. The low-elevation-angle array antenna had linear polarization. The satellite handover problem between the two antennas was substantial.)

- Will operate at all GNSS frequencies (1164 to $1610 \mathrm{MHz}$ )

- Sharp antenna cutoff on the horizon for mitigation of ground multipath at low elevation angles

- $\quad 2.5 \mathrm{~dB} /{ }^{\circ}$ gain slope on the horizon

- $\quad 30 \mathrm{~dB}$ sidelobe level at $-6^{\circ}$ elevation angle

- $\quad 30 \mathrm{~dB}$ sidelobes in the lower hemisphere for mitigation of ground multipath at higher elevation angles

- High quality carrier delay (antenna phase center) and code (group) delay characteristics

- $\quad$ rms carrier delay variation $<0.007 \mathrm{~m}$

- $\quad$ rms code delay variation $<0.020 \mathrm{~m}$

- Circular polarization at low elevation angles for mitigation of lateral multipath

- $\quad$ Taxing aircraft, hangars, etc.

- Unit-to-unit de-correlated sidelobes in the lower hemisphere

- Enhances ground multipath de-correlation amongst ground reference receivers

- Integral air terminal for lightning protection

- Non-scattering air terminal configuration, no performance degradation

As of January 2009, 62 ARL-1900 antennas had been manufactured and tested. Local-area augmentation system/ground-based augmentation system GPS landing systems equipped with ARL1900 antennas have been installed at Atlantic City, NJ; Memphis, $\mathrm{TN}$; and Bremen, Germany. These systems have been used in operational trials.

The basic concepts presented in this paper, and some implementation details of the array antenna are described in US Patents $5,534,882$ [10] and 6,201,510 [11].
Many thanks are given to Mr. John Warburton (FAA William J. Hughes Technical Center) for his help over the many years with the development of the ARL-1900 antenna. The successful development of the ARL-1900 antenna is credited in great part to the technical leadership of Mr. Gary A. Schay (BAE Systems) and the managerial leadership of Mr. Gary T. Nolan (BAE Systems). Many thanks are also given to the BAE Systems engineering, technical support, and management team that over a span of more than 10 years brought the ARL-1900 antenna to fruition.

\section{Appendix: Definition of Antenna Phase Center}

IEEE 100, The Authoritative Dictionary of IEEE Standards Terms, Seventh Edition, provides the following definition:

\begin{abstract}
phase center The location of a point associated with an antenna such that, if it is taken as the center of a sphere whose radius extends into the far-field, the phase of a given field component over the surface of the radiation sphere is essentially constant, at least over that portion of the surface where the radiation is significant. Note: Some antennas do not have a unique phase center.
\end{abstract}

The definition below has been used to quantify the phase characteristics of GPS antennas. (For the ideal case of a point-phasecenter antenna, both definitions yield the same result.)

average phase center The location of the phase reference point associated with an antenna that minimizes the far-field phase variation of a given field component over a given antenna coverage region. Note: Every antenna has a unique average phase center.

The phase variation over the antenna's coverage region can be measured with respect to the average phase center and then used to calibrate the GPS antenna. For precise surveying (mm accuracy), the calibration data is used to correct satellite carrier-delay measurements, depending on the position of the satellite within the antenna's coverage region.

(It is possible to define a code (group) delay reference point:

group delay center The location of the group delay reference point associated with an antenna that minimizes the far-field variation of a given group delay component over a given antenna coverage region.

This point is not necessarily the same point as the average phase center. It has been found that for a well-designed antenna, the average phase center and the group-delay center are closely related. For all practical purposes, there is no need for using another spatial reference point other than the average phase center.) 


\section{References}

1. M. Kayton, "Navigation Land, Sea, Air, \& Space," New York, IEEE Press, 1990, p. 237.

2. FAA Web site, "Local Area Augmentation System - How It Works," available at http://www.faa.gov/about/office org/ headquarters_offices/ato/service_units/techops/navservices/gnss/ laas/howitworks/.

3. A. R. Lopez, "LAAS/GBAS Ground Reference Antenna with Enhanced Mitigation of Ground Multipath," Proceedings of the Institute of Navigation National Technical Meeting, January 2008; http://www.arlassociates.net/2008GPSAntGrndReflect.pdf.

4. A. R. Lopez, "LAAS Reference Antennas - Circular Polarization Mitigates Multipath Effects," Proceedings of the Institute of Navigation Annual Meeting, June 2003; http:// www.arlassociates.net/2003GPSAntCPEffects.pdf.

5. A. R. Lopez, "Calibration of LAAS Reference Antennas," Proceeding of the 14th International Technical Meeting of the Satellite Division of the Institute of Navigation, September 2001; http:// www.arlassociates.net/2001GPSAntCalibration.pdf.

6. FAA, "Lightning and Surge Protection, Grounding, Bonding and Shielding Requirements for Facilities and Electronic Equipment," FAA-STD-019d, August 9, 2002.

7. A. R. Lopez, "Sharp Cutoff Radiation Patterns," IEEE Transactions on Antennas and Propagation, AP-27, 6, November 1979, pp. 820-824; http://www.arlassociates.net/ Nov1979ArraySharpCutoff.pdf.

8. A. R. Lopez, "GPS Reference Antennas for Local Augmentation System, LAAS," Proceedings of the National Technical Meeting of The Institute of Navigation, January 2000; http:// www.arlassociates.net/2000GPSAnt.pdf.

9. M. S. Braasch, "Multipath Effect," in B. W. Parkinson and J. J. Spilker (eds.), Global Positioning Theory and Application, Volume 1, Reston, VA, AIAA, 1996, Chapter 14, pp. 560-566.
10. A. R. Lopez, GPS Antenna Systems, US Patent 5,534,882, issued July 9,1996 .

11. A. R. Lopez, R. J. Kumpfbeck, and E. M. Newman, Self-Contained Progressive-Phase GPS Elements and Antennas, US Patent 6,201,510, issued March 13, 2001.

\section{Introducing the Feature Article Author}

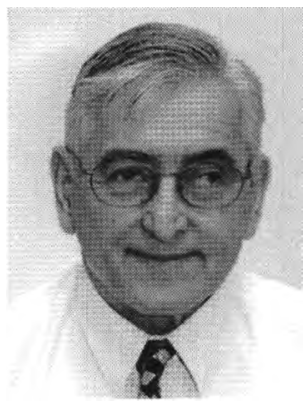

Alfred R. Lopez is an IEEE Life Fellow and a BAE Systems Engineering Fellow. He has 50 years of experience in antenna design, propagation analysis, and the design and development of radiating systems. All of this time he has been with BAE systems, through a heritage linking back to the Hazeltine Corporation and Wheeler Laboratories, where he started his career in 1958. Over most of his career, he has specialized in antenna designs and systems for aircraft-approach and landing operations. He has also contributed to the fields of electronically scanned array antennas, antennas for cellular communications, and ground reference antennas for differential GPS.

In 1958, he received the BEE from Manhattan College, and in 1963, he received the MSEE from the Polytechnic Institute of Brooklyn. He has published more than 60 papers in IEEE and Institute of Navigation publications. He has been awarded 48 US patents, and has received several awards from the IEEE and BAE Systems. 